\title{
ÉTUDE DES POTENTIALITÉS D'ÉLEVAGE INTENSIF DU GOUJON (GOBIO GOBIO, L.) COMPARAISON DE PLUSIEURS TYPES D'ALIMENTS
}

\author{
P. KESTEMONT * \\ Unité d'Ecologie des Eaux Douces (UNECED, Pr. J.-C. MICHA) \\ Facultés Universitaires Notre-Dame de la Paix, 61 rue de Bruxelles, B-5000 NAMUR, Belgique.
}

\section{RESUME}

L'efficacité de différents types d'aliments naturels ou artificiels sur la survie et la croissance des larves de goujon Gobio gobio L. a été étudiée dans deux expériences. II s'est avéré qu'une alimentation du type des nauplii d'Artemia salina congeles permet d'obtenir de bons résultats pendant les deux premiers mois d'élevage. Après 60 jours de nourrissage, la survie des larves est supérieure à $80 \%$ et leur taille moyenne atteint $30 \mathrm{~mm}$. Après la $3^{\mathrm{e}}$ semaine, une alimentation artificielle du type Trouvit starter (0OO) peut remplacer efficacement la nourriture naturelle à la condition que les alevins soient progressivement accoutumés à leur nouvel aliment.

\section{SUMMARY}

\section{ON THE POTENTIALITIES OF GUDGEON INTENSIVE CULTURE (GOBIO GOBIO L.). COMPARISON OF DIFFERENT KINDS OF FOOD.}

The suitability of some different natural and artificial feedstuffs on the survival and growth of fry of gudgeon (Gobio gobio L.) was investigated in two experiments. It was found that frozen nauplii of $A$. salina gave good results during the first two months of rearing. After 60 days, the survival of the fish is higher than $80 \%$ and the mean length reaches $30 \mathrm{~mm}$. After the third week, dry feed (Trouvit starter. 000 ) can take the place of natural food, provided that the fry are accustomed to their new kind of food.

\section{INTRODUCTION}

La pêche sportive est devenue, en Belgique comme dans de nombreux autres pays d'Europe, une activité très répandue. Le nombre de pêcheurs en Europe, à l'exception de l'U.R.S.S., était en 1976 de plus de 16 millions (EIFAC, 1976) et leur nombre était en augmentation rapide (8-16\% par an). La Belgique compte, à elle seule, plus de 250.000 pêcheurs. Parmi les différentes méthodes de pêche à la ligne, la pêche aux vifs est sans aucun doute une des plus appréciées. Face aux demandes souvent insatisfaites en poissons-appâts, certains pisciculteurs belges importent, par centaines de milliers, des vifs venant de l'étranger (notamment des minnows américains du genre Pimephales). II nous a donc semblé intéressant de tenter l'élevage d'une espèce locale répondant aux critères exigés pour les poissons-appâts (DOBIE et al., 1956), ce qui est le cas du goujon (Gobio gobio, L.).

Quelques aspects de la biologie du goujon (saison de reproduction, alimentation, croissance) ont été présentés par BERNET (1960), KENNEDY et FITZMAURICE (1972), BRUNET et HOESTLANDT (1972). Des études plus approfondies sur le développement larvaire ont été réalisées par KENNEDY et FITZMAURICE (1972) et PENAZ et PROKES (1978, 1979). BRUNET et HOESTLANDT (1972) rapportent une série d'expériences de nourrissage à base d'alimentation naturelle et artificielle, mais la croissance des alevins reste généralement très faible et ne dépasse pas $14 \mathrm{~mm}$ après 75 jours d'élevage. De plus, les mortalités sont très importantes, avec une survie maximum de $25 \%$ en conditions expérimentales. Ils préconisent une nourriture vivante, telle que des daphnies, dont les normes d'élevage sont bien connues (DE PAUW et al., 1981). Plusieurs travaux (ROYAUX, 1976 ; LAMBLOT, 1977 ; CHAPEAU, 1983) réalisés au sein de l'Unité d'Ecologie des Eaux Douces ont procuré un certain nombre d'informations sur la biologie de l'espèce et sur ses perspectives d'avenir en pisciculture. CHAPEAU (1983) obtient plusieurs pontes " naturelles "en aquarium et effectue plusieurs pontes artificielles, ce qui lui permet d'effectuer différents essais de nourrissage à partir de jeunes larves de goujon.

Les expériences décrites ci-après sur l'influence de différents types d'aliments sur le pourcentage de survie et la croissance des alevins de goujons s'intègrent dans une étude globale visant à maîtriser et à optimiser la reproduction et l'élevage de l'espèce.

\section{1 - MATÉRIEL ET METTHODES}

\section{Dispositif expérimental (fig. 1)}

La première expérience est réalisée dans une série de 20 petits aquariums en P.V.C., $(0,19 \mathrm{~m} \times$ 


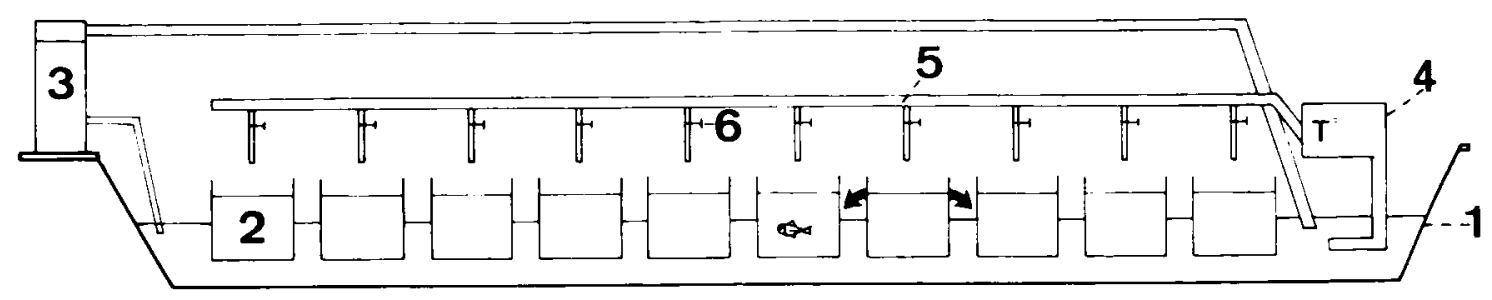

Figure 1: Dispositif expérimental de nourrissage d'alevins.

Batterie de 20 aquariums $(3$ ) disposés en 2 colonnes.

Système d'alimentation en eau permettant un débit réglable dans les différents aquariums.

1. Bassin d'élevage en P.V.C. (Purewell, G2) ; 2. Aquariums de nourrissage : 3. Filtration ; 4. Pompe chauffante; 5 . Alimentation en eau; 6 . Vanne de réglage du débit.

Figure 1: Experimental installation for fry feeding.

Twenty aquaria ( 3 ) ) arranged in two columns.

Water supply with a regulated flow in the different aquaria.

1. Rearing tank in P.V.C. (Purewell, G2) : 2. Aquaria for feeding ; 3. Filtration :

4. Heater pump; 5 . Water supply; 6 . Flow water-gate.

$0.13 \mathrm{~m} \times 0.12 \mathrm{~m}$ ) d'une contenance de 3 litres, disposés en batterie dans un bassin classique d'alevinage à truite de $2,74 \mathrm{~m}$ de long sur $0,45 \mathrm{~m}$ de large et $0,23 \mathrm{~m}$ de haut (PUREWELL, G2). La deuxième expérience utilise des aquariums plus grands $(0,25 \mathrm{~m} \times 0,14 \mathrm{~m} \times 0,17 \mathrm{~m})$ d'une contenance de 6 litres. Les aquariums sont équipés d'un système de trop-plein, avec tamis de $250 \mu$, permettant à l'eau de s'écouler tout en empêchant le passage aux alevins.

La distribution d'eau est effectuée de façon régulière et constante dans les différents bacs grâce à un système de réglage du débit assurant un renouvellement d'eau identique dans chaque aquarium. L'alimentation en eau est assurée par une pompe chauffante, à débit de $13 \mathrm{l} / \mathrm{min}$ (thermomix, 1441), maintenant une température constante de $20^{\circ} \mathrm{C}$ pendant toute la durée de l'expérience. Après passage dans les aquariums, l'eau est récupérée dans le bac d'élevage et filtrée (filtre EHEIM, 1021) afin de lui maintenir une qualité adéquate.

\section{Source d'alevins}

Les géniteurs ont été capturés par pêche électrique le 16 mai 1984 dans le Samson, petit affluent salmonicole de la Meuse. La reproduction artificielle des individus capturés fut réalisée suite à l'injection d'extraits hypophysaires bruts de carpe (Hydroquest International Corporation), à raison de $10 \mathrm{mg} / \mathrm{kg}$ pour les femelles et $3 \mathrm{mg} / \mathrm{kg}$ pour les mâles, suivant les techniques développées précédemment pour d'autres espèces (WOYNAROVICH, 1969; CHAUDHURI, 1976). Après une période moyenne d'incubation, sur claies horizontales, de $115^{\circ}$ jours à $20 \pm 1^{\circ} \mathrm{C}$ et 3 jours de résorption de la vésicule vitelline, les larves furent placées dans les conditions requises pour l'expérience. Un millier d'alevins, provenant de 4 femelles, ont été divisés en 20 groupes de 50 individus, soit une densité de 20 individus par litre.

\section{Expériences d'alimentation}

Le but de la première expérience est de tester différents régimes alimentaires à partir de nourriture naturelle ou artificielle. Les différents aliments utilisés sont Trouvit starter 000 (particules de 100 à $200 \mu$ (Trouw \& Co). Mikromin (particules de 50 à $150 \mu$ )(Tetra Werke). Infusyl (pastilles à bases d'infusoires) (Lab. Appl. Biol. et chim.), Artemia salina L. et Daphnia magna Strauss. Pour chaque type de nour riture, l'expérience est repliquée 4 fois. La nourriture est distribuée en excès 2 fois par jour $(9 \mathrm{~h}$ et $17 \mathrm{~h}$ ). Nous entendons par distribution en excès l'apport de la quantité de nourriture ingérée par les alevins en $1 \mathrm{~h}$, additionnée ensuite d'une faible quantité supplémentaire. L'expérience a été poursuivie pendant 8 semaines, à une température de $20 \pm 1^{\circ} \mathrm{C}$ et sous photo-période naturelle. La mortalité a été enregistrée tous les jours dans chaque aquarium ; la taille individuelle n'a été mesurée qu'après 60 jours.

En ce qui concerne la nourriture vivante, les nauplii d $A$. salina étaient distribués sous forme congelée, après incubation des cystes pendant $24 \mathrm{~h}$ à $32^{\circ} \mathrm{C}$ et sous éclairage et aération continus. Seules les larves d'A. salina écloses étaient distribuées, et non les capsules ou les larves non écloses qui risquent de provoquer des lésions internes entrainant la mort des alevins. Un stock de daphnies vivantes fut constitué pendant les mois qui ont précédé l'expérience, suivant le procédé utilisé par le 
Laboratoire de Mariculture de l'Université d'Etat à Gand (De PAUW et al., 1981). Avant le nourrissage des larves, les daphnies étaient filtrées sur tamis de $250 \mu$ pour ne récupérer que les jeunes individus.

L'expérience 2 a pour but de déterminer le moment à partir duquel une alimentation artificielle peut remplacer efficacement une alimentation naturelle. Six cents alevins sont séparés en 6 groupes de 100 individus, à une densité égale à celle de la première expérience, soit 20 ind. /litre. Les conditions de température, d'oxygénation et de renouvellement d'eau sont identiques à celles de l'expérience 1 . Chaque traitement est repliqué 2 fois. Un premier groupe reçoit des $A$. salina pendant 6 semaines (témoin), un second groupe reçoit des $A$. salina pendant 3 semaines et ensuite du Trouvit OOO, et un troisième est accoutumé à la nourriture artificielle par apport simultané, entre la troisième et la quatrième semaine, de Trouvit 000 et d'A. salina avant de recevoir cette nourriture artificielle.

\section{Analyse des données}

L'ensemble des résultats sont traités par une analyse de la variance à un critère de classification, ANOVA 1 (DAGNELIE, 1975). Un test de Bartlett (DAGNELIE, 1975) fut, au préalable, réalisé afin de vérifier l'homogénéité des variances. Sur base des résultats de l'ANOVA 1, une série de contrastes orthogonaux nous permit de comparer dans la première expérience, la taille des alevins suite aux différents traitements alimentaires, et, dans la deuxième expérience, l'efficacité d'une modification du type de nourriture. Les valeurs de taille furent préalablement transformées en logarithmique népérien $(1 n)$.

\section{2 - RESUltats}

Le dispositif expérimental a fonctionné de façon satisfaisante pendant toute la durée des expériences. Un nettoyage quotidien des aquariums était cependant conseillé afin de récupérer les restes de nourriture non ingérée qui sédimentaient et constituaient des substrats favorables au développement des saprolégniées et des bactéries.

\section{Expérience 1}

Le nombre et le pourcentage de survie observés pour chaque type d'aliment, dont l'évolution est présentée aux Tabl. 1 et Fig. 2, peuvent être légèrement augmentés (environ 5 à $10 \%$ ) étant donné les manipulations de comptage qui furent une des causes de mortalité des alevins.

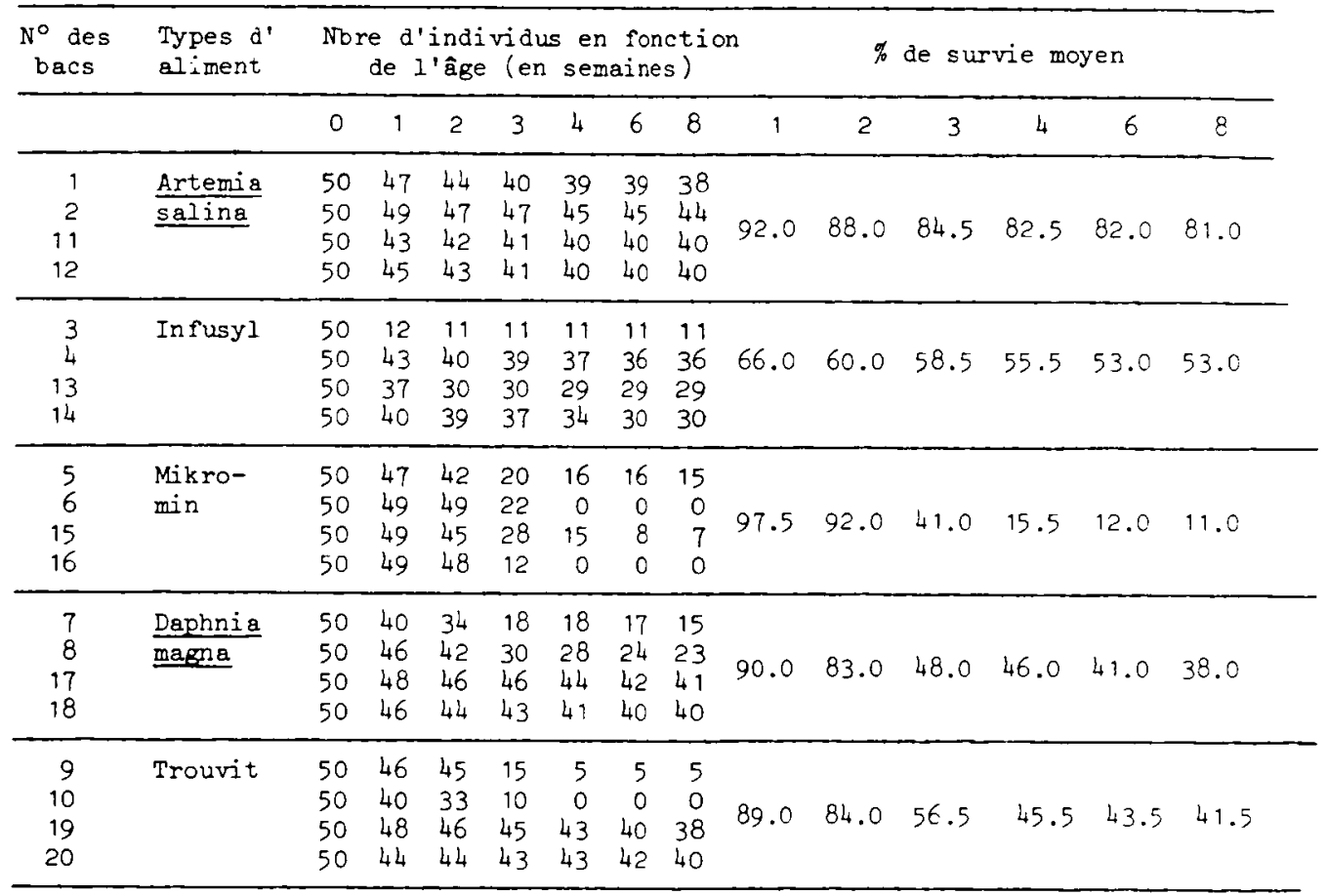

Tableau 1 : Evolution du nombre et du pourcentage de survie d'alevins de goujon (Gobio gobio, L.) alimentés différemment.

Table 1: Evolution of the number and percentage of gudgeon fry survival (Gobio gobio, L.) fed with different kinds of food. 


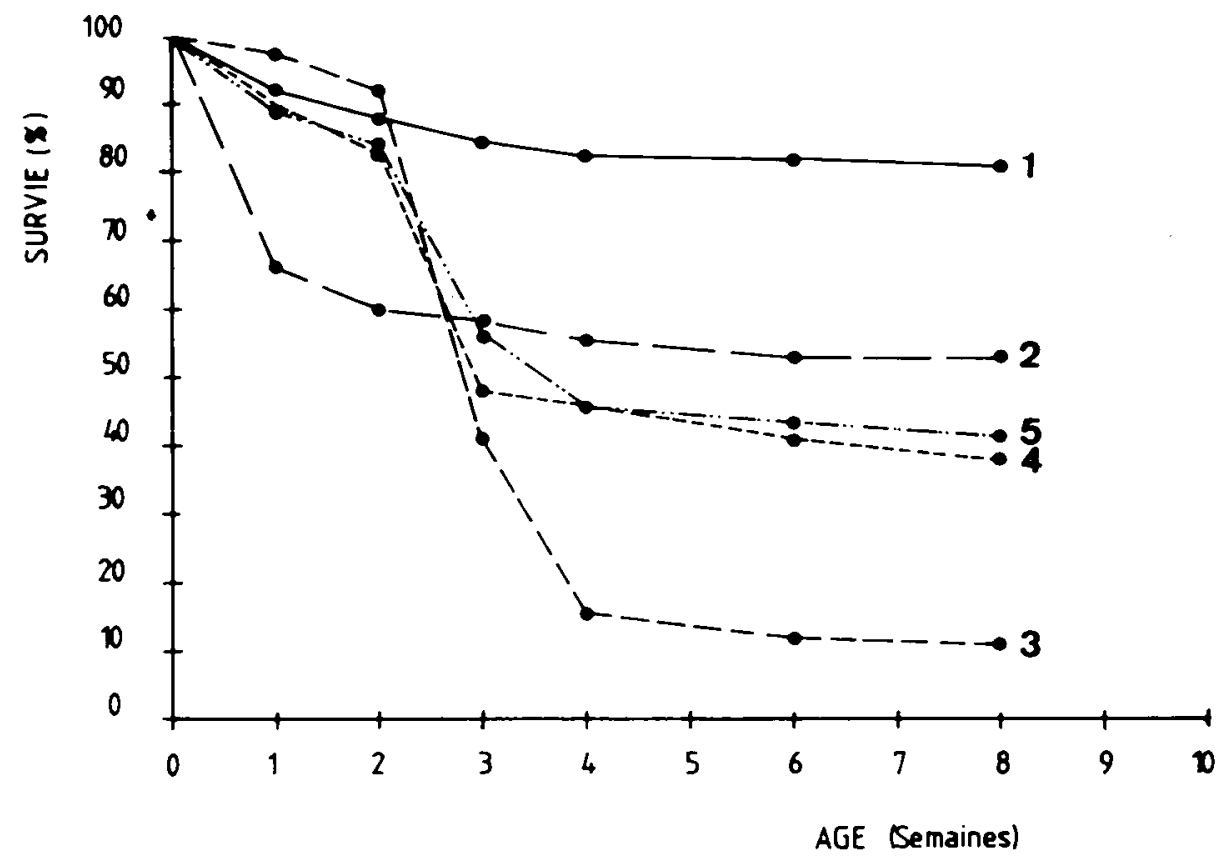

Figure 2 : Évolution de la survie de larves (Gobio gobio) (L) en fonction de l'alimentation pendant les 8 premières semaines.

1. A. salina : 2. Infusyl ; 3. Mikromin : 4. Daphnia magna ; 5. Trouvit 000

Figure 2: Evolution of fry survival (Gobio gobio, L.) in function of feeding, during the first eight weeks.

1. A. salina ; 2. Infusyl ; 3. Mikromin : 4. Daphnia magna ; 5. Trouvit 000

A. salina maintient, jusqu'à l'âge de 8 semaines, un pourcentage de survie constant et élevé dans les différents bacs. Le Trouvit 000 permet d'obtenir, pour 2 bacs sur les 4 , des pourcentages très satisfaisants, de 76 à $80 \%$, ce qui apparaît évidemment très intéressant, vu le prix relativement bas de l'aliment, mais les restes de Trouvit non assimilés permettent le développement très important de saprolégniées ayant entraîné une mortalité rapide dans les 2 autres bacs. De plus, les alevins montrent une croissance relativement lente, nettement inférieure à celle obtenue avec $A$. salina (Tabl. 2). De même, les alevins nourris à partir de daphnies grandissent lentement, suite à la trop grande taille de $D$. magna pendant les premières semaines de vie des larves de $G$. gobio. Les alevins nourris au Mikromin qui, pendant les deux premières semaines, présentaient les meilleurs pourcentages de survie (aliment micronisé, facilement accessible aux jeunes larves) ont ensuite connu des mortalités très importantes. La grande variance observée au sein de certains traitements alimentaires ne nous a cependant pas permis de réaliser une ANOVA 1 , suite au rejet du critère d' homogénéité des variances (test de Bartlett).

La taille des goujons, au terme de l'expérience, est présentée au Tabl. 2. La densité, égale en début d'expérience, a varié d'un facteur 7 en fin d'expérimentation. L'ANOVA 1 , à partir des données transformées en logarithme indique un effet aliment hautement significatif $(P<0,01)$ sur la taille des alevins $(F=171.08)$. Les contrastes réalisés entre $A$. salina et les autres aliments révèlent une taille significativement plus grande $(P<0,01)$ lorsque les alevins sont nourris à partir d'A. salina.

\section{Expérience 2}

Les résultats de la survie des alevins de goujon ayant subi une modification dans le type d'aliments après la $3^{e}$ semaine sont présentés au Tabl. 3 . La survie dans les groupes I et lli reste élevée en fin d'expérience alors qu'une brusque modification dans l'alimentation a été mal supportée par les alevins du groupe II. L'ANOVA 1 indique un effet traitement hautement significatif $(F=142,78 ; P<0,01)$. Les contrastes orthogonaux (Tabl. 4) réalisés entre les régimes alimentaires montrent qu'il n'y a pas de différence significative entre le groupe l et le groupe III. La survie est par contre significativement plus faible lorsqu'il n'y a pas de période de transition entre les 2 types d'alimentation.

En fin d'expérience, les alevins ont été placés en aquarium de $100 \mathrm{I}$, à une densité de 4 ind. / et 


\begin{tabular}{|c|c|c|c|c|c|c|}
\hline$N^{\circ}$ bacs & Aliment & $\begin{array}{l}\text { Nore } \\
\text { initial }\end{array}$ & $\begin{array}{l}\text { Nore } \\
\text { final }\end{array}$ & $\begin{array}{l}\text { Dens . moy. } \\
\text { finale } \\
\text { (ind./1.) }\end{array}$ & $\begin{array}{l}\text { L.T. moy. } \\
(\mathrm{mm}) \\
(\mathrm{I} . \mathrm{C} .)\end{array}$ & $\begin{array}{l}\text { Contr. orthog. } \\
\text { Artemia }\end{array}$ \\
\hline $\begin{array}{ll}1,2 \\
11, & 12\end{array}$ & $\frac{\text { Artemia }}{\text { salina }}$ & 200 & 162 & 13.0 & $30.0( \pm 0.89)$ & - \\
\hline $\begin{array}{ll}3,4 & \\
13, & 14\end{array}$ & Infusy I & 200 & 106 & 8.5 & $22.7( \pm 0.91)$ & $9.294^{x *}$ \\
\hline $\begin{array}{l}5,6 \\
15,16\end{array}$ & Mikromin & 200 & 22 & 1.8 & $21.0( \pm 0.72)$ & $11.667^{x x}$ \\
\hline $\begin{array}{l}7,8 \\
17,18\end{array}$ & $\frac{\text { Daphnia }}{\text { magna }}$ & 200 & 117 & 9.4 & $15.0( \pm 0.65)$ & $22.518^{* x}$ \\
\hline $\begin{array}{l}9,10 \\
19,20\end{array}$ & Trouvit & 200 & 83 & 6.6 & $15.9( \pm 0.92)$ & $21.192^{x x}$ \\
\hline
\end{tabular}

Tableau 2 : Taille des alevins de goujon Gobio. gobio L. après 60 jours d'alimentation par differents types d'aliment.

Dens. moy. = densité moyenne

L.T. moy. = longueur totale moyenne

I.C. = intervalle de confiance

$x \times p<0.01$

Contr. Orthog. $=$ contrastes orthogonaux

Table 2 : Length of gudgeon fry, Gobio gobio L., after 60 days of feeding with different kinds of food.

Dens. moy. = Mean density

L.T. moy. = Total mean length

I.C. = confidence level

$x x p<0,01$

Contr. Orthog. = orthogonal contrasts.

à une température constante de $20^{\circ} \mathrm{C}$. Le Trouvit représente alors l'aliment unique et est distribué 2 fois par jour ( $9 \mathrm{~h}$ et $17 \mathrm{~h}$ ), 6 jours par semaine. La mortalité est négligeable ( $3 \%$ ) au bout du $3^{\mathrm{e}}$ mois et la taille moyenne des individus atteint $41 \mathrm{~mm}$, avec des extrêmes de 36 à $47 \mathrm{~mm}$.

\begin{tabular}{clccc}
\hline Nobacs & Aliments & $\begin{array}{l}\text { Nbre } \\
\text { initial }\end{array}$ & $\begin{array}{c}\text { Nore } \\
\text { final }\end{array}$ & $\begin{array}{c}\text { \% survie moyen } \\
\text { après } 6 \text { semaines }\end{array}$ \\
\hline I & Artemia & 100 & 85 & $84.0 \%$ \\
I & & 100 & 83 & \\
II.1 & Artemia puis & 100 & 61 & \\
II.2 & Trouvit & 100 & 60 & $60.5 \%$ \\
III.1 & Artemia puis & 100 & 80 & \\
III.2 & Artemia + & 100 & 83 & $81.5 \%$ \\
& Trouvit puis & & & \\
\hline
\end{tabular}

Tableau 3 : Pourcentage de survie ('alevins de goujons (Gobio gobio, L.) en fonction de l'alimentation. Modificatic du régime alimentaire après la 3ème semaine.

Table 3 : Percentage of gudgeon:- survival (Gobio gobio, L.) in function of feeding. Modification in the diet after the t.ird week. 


\begin{tabular}{cccc}
\hline & $\begin{array}{c}\text { Artemis } \\
\text { Auis } \\
\text { Trouvit }\end{array}$ & $\begin{array}{c}\text { Artemia } \\
\text { Artemia + Trouvit } \\
\text { Trouvit }\end{array}$ & T obs \\
\hline+1 & 0 & -1 & $1.636^{\circ}$ \\
+1 & -1 & 0 & $15.384^{\times *}$ \\
0 & +1 & -1 & $13.748^{x \times}$ \\
\hline
\end{tabular}

Tableau 4 : Comparaison de la survie d'alevins de goujons, (Gobio gobio, L.), suite à une modification du régime alimentaire. Résultats des contrastes orthogonaux effectués entre les différents traitements.

10 : non significatif

Xx : significatif

$P<0,01$ ).

T. Obs. : valeur observée de $t$ (Student)

Table 4 : Comparison of the survival of gudgeon fry, (Gobio gobio, L.), following a modification in the diet.

Results of the orthogonal contrasts between the different treatments.

10 : non significant

$x x$ : significant $P<0,01$ ).

T. Obs. : t-test observed.

\section{3 - DISCUSSION}

D'après nos résultats et dans les conditions d’élevage où nous avons opéré, il est clair qu'un apport d'aliments concentrés secs ne peut être recommandé pour les premiers stades de développement des larves de goujon. Par contre, une nourriture naturelle du type des nauplii d'A. salina, même sous forme congelée, permet d'obtenir les meilleurs pourcentages de survie (supérieurs à $80 \%$ ), associés à une taille maximale après 2 mois d'élevage. Ces résultats sont en accord avec ceux obtenus par HOGENDOORN (1980) sur l'élevage du silure africain, Clarias lazera C. \& V. Une optimisation de la méthode pourrait être envisagée grâce à une alimentation à partir d'A. salina vivantes (et non congelées) et une étude des effets de variations sur le niveau et la fréquence alimentaires (BRYANT et MATTY, 1980 ; HOGENDOORN, 1980).

CHIBA (1961) indique que les larves de carpe commune nourries à partir de Daphnia carinata, à une température de $18-23^{\circ} \mathrm{C}$ augmentent entre 2 et 3 fois leur poids chaque jour. L'alimentation à base du cladocère Moina spp. (BARANOVA, 1974) permet également une croissance similaire des jeunes carpes pendant les 10 premiers jours. Nos résultats tendent cependant à sous-estimer l'efficacité des cladocères, du moins du type $D$. magna, pendant les premières semaines d'élevage, suite à la trop grande taille des organismes distribués.

Après la troisième semaine, l'apport du Trouvit 000 semble très bien accepté par les alevins de goujon, pour autant que la nourriture artificielle soit distribuée, pendant plusieurs jours, au moins, en mélange avec des Artemia. Un changement brusque dans le type d'alimentation n'est pas accepté directement par les alevins qui ne se nourrissent pas et dont la mortalité est importante dans les premiers temps. A la fin du premier mois, le Trouvit peut donc représenter l'aliment unique pour les jeunes goujons et permet une croissance rapide des individus qui peuvent dépasser $45 \mathrm{~mm}$ à l'âge de 3 mois.

Aucune attention n'a été portée, dans ce travail, pour rechercher les effets de la température et de la densité d'élevage sur la croissance des larves. Il faut cependant noter que le renouvellement d'eau était suffisant pour maintenir une qualité d'eau acceptable. A titre d'indication, SYAZUKI et HAYAMA (1957) montrent d'ailleurs qu'à densité de stockage raisonnable, un renouvellement d'eau 
excédant les besoins en $\mathrm{O}_{2}$ des larves de carpe suffit à prévenir toute réduction dans la vitesse de croissance. D'autre part, KOSSMAN (1976) signale que, dans l'écloserie d'Ahrensbrug, les larves de carpes sont élevées à raison de 50.000 par aquarium de 40 / (plus de 1.200 ind. /1). Un renouvellement d'eau d'un $\mathrm{I} / \mathrm{mn}$ est suffisant pour assurer l'oxygénation d'une telle installation.

\section{REMERCIEMENTS}

Je tiens à exprimer ma gratitude au Prof. J. - C. MICHA, pour l'aide apportée dans la réalisation des expériences et dans la rédaction de cet article. Je remercie également le Dr. N. De PAUW. du Laboratoire de Mariculture de l'Université d'Etat à Gand, de m'avoir initié à l'élevage en masse de Daphnia magna ainsi que le Dr C.J.J. RICHTER pour l'accueil et les conseils judicieux qu'il m'a offerts lors de mon séjour au département de pêche et pisciculture de l'Université Agronomique à Wageningen (Pays-Bas).

\section{REFERENCES}

BARANOVA V.P., 1974. Dépendence of the carp larvae growth rate on condition of rearing. $/ 2 \mathrm{~V}$. Gos. NIORCH., $92: 66-78$.

BERNET B., 1960. Recherches biologiques sur les populations de Gobio gobio, L. de la Nivelle. Ann. Stn. Cent. Hydrob. Appl., $8: 127-180$.

BRUNET R., HOESTLANDT H., 1972. Recherches biologiques et pisciculture expérimentale du goujon (Gobio gobio. L. ). Bull. Franc. Piscicult., 45 (246) : 7-32.

BRYANT P.L., MATTY A.J., 1980. Optimisation of Artemia feeding rate for carp larvae. Aquaculture. $21: 203-212$.

CHAPEAU J.P., 1983. Contribution à l'étude des potentialités d'élevage du goujon Gobio gobio, L. Mémoire U.C.L. Inédit, 126 p.

CHAUDHURI H., 1976. Use of hormones in induced spawning of carp. J. Fish. Res. Board. Can., 33. 940-947.

CHIBAK., 1961. The effect of food quantity and quality on the survival and growth of common carp fry. Bull. Freshwater Res. Lab., $11: 105-132$.

DAGNELIE P., 1975 : Théorie et méthodes statistiques. Vol. 2. 463 p. Presses Agronomiques de Gembloux.

DE PAUW N., LAUREYS P., MORALES J.. 1981. Mass cultivation of Daphnia magna Strauss on ricebran. Aquaculture, $25: 141-152$.

DOBIE J. et al., 1956. Raising bait fishes. U.S. Fish and Wildlife Service. Circular 35, 124 p.

EIFAC, 1976. Rapport et documents techniques de la deuxième consultation européenne sur l'évaluation économique de la pêche sportive et commerciale, 22-24 septembre 1975, Göteborg, Suède, EIFAC Tech. Pap., 26, 186 p.

HOGENDOORN H., 1980. Controlled propagation of the african catfish, Clarias lazera (C et V). III. Feeding and growth of fry. Aquaculture, $21: 233-241$.

KENNEDY M.P., FITZMAURICE P., 1972. Some aspects of the biology of gudgeon (Gobio gobio, L.) in Irish waters. J. Fish. Biol., 4. 425-448.

KOSSMAN H., 1976. Rearing of carp fry under laboratory conditions. EIFAC Tech. Pap. $25: 122-126$.

LAMBLOT G.. 1977. Contribution à l'étude de la croissance et de la reproduction d'un poisson cyprinidae, Gobio gobio. L. Mémoire F.U.N. Inédit 105 p.

PENAZ M., PROKES M., 1978. Reproduction and early development of the gudgeon (Gobio gobio, L.) 1. Spawning and embryonic periods. Zool. Listy. 27, 3, 257-267.

PENAZ M., PROKES M., 1979. Reproduction and early development of the gudgeon (Gobio gobio. L.). 2. Larval and juvenile periods. Folia. Zool., Brno, 28, $1: 55-64$.

ROYAUXA., 1976. Contribution à l'étude de la biologie et de la reproduction du goujon Gobio gobio, L. Mémoire F.U.N. Inédit, 127 p.

SYAZUKI K., HAYAMA K., 1957. Studies on the nutrition of carp fry. 3. Consumption of oxygen. J. Shimonoseki Coll. Fish., $6: 255-260$

WOYNAROVICH, 1969. Techniques of hypophysation of common carp. FAO/FUNDP Reg. Seminar on induced breeding of cultivated fishes, Calcutta-Bombay.

YASHOUV A., 1970. Propagation of chironomid larva as food for fish fry. Bamidgeh, 22, $4: 101-105$. 